Correspondence

Wen-Chien Ko

winston@mail.ncku.edu.tw

Received 19 September 2011 Accepted 8 February 2012

\section{Distribution and phenotypic and genotypic detection of a metallo- $\beta$-lactamase, CphA, among bacteraemic Aeromonas isolates}

Chi-Jung Wu, ${ }^{1,2,3}$ Po-Lin Chen, ${ }^{1,3}$ Jiunn-Jong Wu, ${ }^{4}$ Jing-Jou Yan, ${ }^{5}$ Chin-Chi Lee, ${ }^{3,6}$ Hsin-Chun Lee, ${ }^{3,6}$ Nan-Yao Lee, ${ }^{3,6}$ Chia-Ming Chang, ${ }^{3,6}$ Yu-Tzu Lin, ${ }^{2}$ Yen-Cheng $\mathrm{Chiu}^{3}$ and Wen-Chien $\mathrm{Ko}^{3,6}$

${ }^{1}$ Department of Graduate Institute of Clinical Medicine, National Cheng Kung University Medical College, Tainan, Taiwan, ROC

${ }^{2}$ National Institute of Infectious Diseases and Vaccinology, National Health Research Institutes, Tainan, Taiwan, ROC

${ }^{3}$ Department of Internal Medicine, National Cheng Kung University Medical College and Hospital, Tainan, Taiwan, ROC

${ }^{4}$ Department of Medical Laboratory Science and Biotechnology, National Cheng Kung University, Tainan, Taiwan, ROC

${ }^{5}$ Department of Pathology, National Cheng Kung University Medical College and Hospital, Tainan, Taiwan, ROC

${ }^{6}$ Center for Infection Control, National Cheng Kung University Hospital, Tainan, Taiwan, ROC

The objectives of the study were to investigate the distribution of $c p h A$-related genes (cphA) encoding a CphA metallo- $\beta$-lactamase (MBL) among 51 consecutive Aeromonas blood isolates and to compare different phenotypic methods for detecting CphA. The presence of cphA was detected by PCR. Four phenotypic methods, the imipenem-EDTA combined disc test, imipenemEDTA MBL Etest, agar dilution test and modified Hodge test (MHT), were used to detect imipenem susceptibility and MBL production. The results showed that 35 (69\%) blood isolates had cphA. All (100\%) of 16 Aeromonas aquariorum isolates and 12 Aeromonas veronii isolates, and $4(80 \%)$ of 5 Aeromonas hydrophila isolates, carried $c p h A$, but none of 15 Aeromonas caviae isolates did. With the standard inocula, irrespective of the presence or absence of $c p h A$, all but one $(50,98 \%)$ isolates were susceptible to imipenem tested by disc diffusion, Etest and agar dilution ( $10^{4}$ c.f.u. spot inocula), and did not exhibit MBL production by the imipenem-EDTA combined disc test and MBL Etest. By the agar dilution test using large inocula $\left(10^{7}\right.$ c.f.u.), 34 (97\%) of $35 c p h A^{+}$isolates had imipenem MICs of $\geqslant 16 \mu \mathrm{g} \mathrm{ml}^{-1}$, higher than the susceptible breakpoint $\left(4 \mu \mathrm{g} \mathrm{ml}^{-1}\right.$ ), and demonstrated positive results for the MHT, while one $c p h A^{+}$and all $17 c p h A^{-}$isolates had imipenem MICs of $\leqslant 4 \mu \mathrm{g} \mathrm{ml}^{-1}$. In conclusion, the distribution of cphA among aeromonads is species-specific, found in $A$. aquariorum, $A$. veronii and A. hydrophila, and the MHT may be a phenotypic screening test for CphA production.
Abbreviations: CLSI, Clinical and Laboratory Standards Institute; MBL, metallo- $\beta$-lactamase; MHT, modified Hodge test.

The GenBank/EMBL/DDBJ accession numbers for the partial sequences of the $r p o B$ genes of Aeromonas aquariorum MDC47 ${ }^{\top}$ $\left(=\mathrm{BCRC} 17946^{\top}\right)$ and $A$. aquariorum A2-13, A2-42, A2-56, A2-61, A270, A2-94, A2-98, A2-107, A2-155, A2-157 and A2-159 are JF972604-JF972615, and those for the partial sequences of the cphA-related genes of $A$. aquariorum MDC $47^{\top}\left(=\mathrm{BCRC} 17946^{\top}\right)$ and $A$. aquariorum A2-13, A2-42, A2-56, A2-61, A2-70, A2-94, A2-98, A2107, A2-157 and A2-159 are JF972618-JF972628.

A supplementary figure is available with the online version of this paper.

\section{INTRODUCTION}

Aeromonads, oxidase-producing Gram-negative rods, are aquatic micro-organisms and have been implicated in a variety of human diseases, ranging from mild gastroenteritis (Janda \& Abbott, 2010) to fatal septicaemia, peritonitis/abdominal sepsis (Wu et al., 2009) and necrotizing fasciitis (Lee et al., 2008). Three principal classes of chromosomally mediated $\beta$-lactamases have been recognized in Aeromonas species, i.e. Ambler class C cephalosporinases, class D penicillinases and class B 
metallo- $\beta$-lactamases (MBLs) (Janda \& Abbott, 2010). Fosse et al. (2003) characterized the strains producing these $\beta$-lactamases into five major phenotypes: Aeromonas hydrophila complex/class B, C and D $\beta$-lactamases; Aeromonas caviae complex/class $\mathrm{C}$ and $\mathrm{D} \beta$-lactamases; Aeromonas veronii complex/class $\mathrm{B}$ and $\mathrm{D} \beta$-lactamases; Aeromonas schubertii spp./class D $\beta$-lactamase; and Aeromonas trota spp./class C $\beta$-lactamase.

The most commonly found MBL in Aeromonas species is CphA, which was first named in 1991 (Massidda et al., 1991). In addition to CphA, other MBLs have been identified in aeromonads, including ImiS (Walsh et al., 1998), IMP-19 (Neuwirth et al., 2007) and VIM (Libisch et al., 2008). CphA, a metalloenzyme, is inhibited by metal chelators, such as EDTA (Massidda et al., 1991; Segatore et al., 1993). Compared with other class B enzymes, CphA MBL has a very specific substrate profile, being active on penem and carbapenem only, and not on penicillin and cephalosporins (Segatore et al., 1993). The distribution of $\operatorname{cph} A$-related genes is species-related: they are mainly found in isolates of A. hydrophila, A. veronii and Aeromonas jandaei, but not in A. caviae (Balsalobre et al., 2009; Rossolini et al., 1995). However, CphA MBL production is not easily detected by in vitro susceptibility testing unless using large inocula. Tested by the agar dilution method with a larger inoculum $\left(10^{8}\right.$ c.f.u.), the imipenem MICs for Aeromonas strains with $c$ phA-related genes were $\geqslant 4 \mathrm{mg}$ $\mathrm{ml}^{-1}$, higher than the susceptible breakpoint, in a study by Rossolini et al. (1995).

Recent developments in the field of Aeromonas taxonomy based on genetic identification have led to a reclassification of aeromonads and to the description of novel species. For example, several clinical isolates from Taiwan were identified as A. aquariorum (Figueras et al., 2009), a recently described species found in aquarium water and ornamental fish (Martínez-Murcia et al., 2008). Furthermore, A. aquariorum was found to be widely distributed in clinical and environmental specimens, and could be misidentified as $A$. hydrophila (Aravena-Román et al., 2011). A. aquariorum can be associated with diarrhoea, bacteraemia, skin and soft tissue infections, and other extraintestinal infections, and clinicians should be aware of this novel clinically relevant species (Figueras et al., 2009). An understanding of the $\beta$-lactamase types intrinsically harboured in this novel species would be a guide for antimicrobial therapy. However, current knowledge about $c p h A$ distribution in this novel species is limited.

In this work, we investigated the prevalence of $c p h A$-related genes and evaluated different phenotypic methods for detecting CphA MBL in reference strains and blood isolates of different Aeromonas species, trying to expand the knowledge of the occurrence and distribution of CphA MBL and $c p h A$-related genes in the members of the genus Aeromonas, with special attention to the novel species $A$. aquariorum.

\section{METHODS}

Bacterial isolates and species identification. Consecutive Aeromonas blood isolates at National Cheng Kung University Hospital, a medical centre in southern Taiwan, were collected from June 1999 to June 2001 and stored at $-70{ }^{\circ} \mathrm{C}$ until use. Only the first isolate of the same species from each patient was studied. During the study period, 51 blood isolates from 47 patients were available. An Aeromonas blood isolate, A2-1000804, which was collected in 2011 from a patient with a febrile episode while receiving the 7 th day of ertapenem therapy for biliary tract infection caused by Escherichia coli producing extended-spectrum $\beta$-lactamase, was also included. The genus Aeromonas was identified by positive oxidase test, fermentation of D-glucose, motility, the absence of growth in $6.5 \%$ sodium chloride and resistance to the vibriostatic agent O/129 (150 $\mu \mathrm{g})$, and was confirmed by the GNI Plus system (Vitek Systems; bioMérieux) or API 20E system (bioMérieux). Species identification was based on both the Vitek system or API 20E system and sequence analyses of the partial $r p o B$ gene obtained by PCR with the primers Pasrpob-L ( $5^{\prime}$ GCAGTGAAAGARTTCTTTGGTTC-3') and Rpob-R (5'-GTTGCATGTTNGNACCCAT-3') (Küpfer et al., 2006). The sequences of amplified DNA products, around $500 \mathrm{bp}$, were compared with reference sequences available in GenBank (http://www.ncbi.nlm.nih. gov/BLAST/). Based on the $r p o B$ results, isolates with a dissimilarity value $\leqslant 0.9 \%$ for a given type strain were identified to that species (Lamy et al., 2010).

A. aquariorum $\mathrm{MDC}^{\mathrm{T}} 7^{\mathrm{T}}\left(=\mathrm{BCRC} 17946^{\mathrm{T}}\right)$, A. veronii bv. sobria ATCC $9071^{\mathrm{T}}$, A. hydrophila ATCC $7966^{\mathrm{T}}$ and A. caviae ATCC $13136^{\mathrm{T}}$ were used as the reference strains for different Aeromonas species.

Detection of cphA-related genes. The presence of $c p h A$-related genes was detected by both PCR amplification and DNA sequence analyses and the colony hybridization technique. PCR was performed with primers designed according to the A. hydrophila AE036 cphA gene (GenBank accession no. X57102), i.e. ANY-SSD/F (F: 5'GCTTAGAGCTCCTAAGGAGCAAGATGAAAGGTTGG-3'; R: 5' $^{\prime}$ GCATAGGTACCTTATGACTGGGGTGCGGCCTTG-3') (Massidda et al., 1991; Talavera et al., 2006), with similar conditions for the $r p o B$ gene amplification except for the annealing temperature, which was changed to $53{ }^{\circ} \mathrm{C}$. The procedures for colony hybridization followed the manufacturer's instructions (DIG DNA Labelling kit; Roche), in which the hybridization probes were designed from the amplified DNA products of isolates of A. aquariorum A2-5, A. veronii A2-7 and A. hydrophila A2-11, around $720 \mathrm{bp}$. The amplified DNA sequences of these three isolates were $94 \%$ identical to those of the $A$. hydrophila AE036 cphA genes.

All imipenem-non-susceptible isolates were examined for other genes encoding MBLs, including $b l a_{\mathrm{IMP}}$ and $b l a_{\mathrm{VIM}}$ genes by previously described PCR primers for IMP and VIM families and conditions (Balsalobre et al., 2009). Two isolates of Pseudomonas aeruginosa with the $b l a_{\mathrm{VIM}-3}$ gene and two isolates of Klebsiella pneumoniae with the $b l a_{\mathrm{IMP}-8}$ gene were used as positive control strains.

Imipenem susceptibility and expression of MBL. The discs with imipenem $(10 \mu \mathrm{g})$ alone or associated with EDTA $(10 \mu \mathrm{l}, 0.5 \mathrm{M})$, the MBL Etest (BD BIODISK, imipenem 4-256 $\mu \mathrm{g} \mathrm{ml}^{-1}$ and imipenem $1-64 \mu \mathrm{g} \mathrm{ml}^{-1}+$ EDTA) and the agar dilution test (imipenem 0.5$16 \mu \mathrm{g} \mathrm{ml}^{-1}$ ) were used to detect imipenem susceptibility and MBL production. The procedures for the disc diffusion test, Etest and agar dilution test followed the Clinical and Laboratory Standards Institute (CLSI) guidelines (CLSI, 2009, 2010). As recommended by the CLSI, standard inocula of $0.5 \mathrm{McFarland}$ for the disc diffusion test and Etest and spot inocula of $10^{4}$ c.f.u. for the agar dilution test were used. Larger inocula of $1.5 \mathrm{McFarland}$ for the disc diffusion test and Etest and spot inocula of $10^{7}$ c.f.u. for the agar dilution test were also adopted. Interpretation of imipenem susceptibility followed the CLSI 
recommendations for Aeromonas species (CLSI, 2010), and results determined with both standard and large inocula adopted the same interpretative criteria for comparison in this study. Isolates that did not fit the criteria of 'susceptible', and belonged to the categories of 'intermediate' and 'resistant', were classified as being 'non-susceptible'. A $>7 \mathrm{~mm}$ increase in the inhibition zone diameter in the presence of imipenem associated with EDTA in comparison with that of imipenem alone was interpreted as evidence of MBL production (Yong et al., 2002). By MBL Etest, the expression of MBL was reflected by a reduction of imipenem MIC by at least eightfold, the appearance of a phantom zone or deformation of the imipenem ellipse in the presence of EDTA. P. aeruginosa ATCC $27853^{\mathrm{T}}$, negative for MBL, was used as the quality control strain. The modified Hodge test (MHT) followed the CLSI standards of a confirmatory test for carbapenemase production in members of the Enterobacteriaceae (CLSI, 2009), and an ertapenem disc $(10 \mu \mathrm{g})$ was the carbapenem used.

Clinical data. Medical records of patients with bacteraemia due to Aeromonas species during the study period were reviewed. The clinical outcome of patients treated by carbapenems was analysed.

\section{RESULTS}

\section{Species and $c p h A-$ related genes distribution}

By sequence analyses of the partial $r p o B$ genes, 16,11 and 8 isolates were identified as A. aquariorum, A. caviae and $A$. veronii, with a dissimilarity value $\leqslant 0.9 \%$ for the $r p o B$ genes of A. aquariorum DSM $18362^{\mathrm{T}}$ (GenBank accession no. FM210471), A. caviae strain V83 (GenBank accession no. AY851107) and A. veronii strain JF2638 (GenBank accession no. AY851124), respectively. Based on typical phenotypic reactions in the Vitek or API 20E system and $r p o B$ gene sequences with $98.6-98.8 \%$ identity to that of $A$. caviae strain V83, another four isolates were identified as $A$. caviae. Likewise, four isolates were identified as $A$. veronii because their $r p o B$ gene sequences had $98.6-98.8 \%$ identity to that of $A$. veronii strain JF2638 and they showed typical phenotypic reactions, and five isolates were identified as $A$. hydrophila because their rpoB sequences had 98.0-98.6\% identity to that of A. hydrophila ATCC 7966 (GenBank accession no. CP000462). However, species identification of three isolates was not possible due to incompatible results of the phenotypic identification and the $r p o B$ sequencing. Overall, 51 blood isolates were identified as $A$. aquariorum (16, $31 \%)$, A. caviae $(15,29 \%)$, A. veronii (12, $24 \%)$, A. hydrophila (5, 10\%) and Aeromonas species (3, $4 \%)$. Some of the isolates had been described previously (Wu et al., 2007; Figueras et al., 2009). The GenBank accession numbers for the $r p o B$ gene sequences of $A$. aquariorum $\mathrm{MDC} 47^{\mathrm{T}}$ and 11 published A. aquariorum blood isolates are JF972604-JF972615. The A2-1000804 isolate with $99.2 \%$ sequence identity of the $r p o B$ gene to that of A. aquariorum DSM $18362^{\mathrm{T}}$ was determined as $A$. aquariorum.

By PCR amplification and sequence analysis, A. aquariorum $\mathrm{MDC}^{\mathrm{T}} \mathrm{T}^{\mathrm{T}}$, A. aquariorum A2-1000804, A. veronii bv. sobria ATCC $9071^{\mathrm{T}}$, A. hydrophila ATCC $7966^{\mathrm{T}}$ and 35 (69\%) of the 51 blood isolates had $c p h A$-related genes. For the 51 blood isolates, the results of colony hybridization were concordant with those of PCR, except for Aeromonas species A2-84, which was hybridization-negative but PCRpositive. Combining the results of PCR and colony hybridization, all $A$. aquariorum $(16 / 16,100 \%)$ and $A$. veronii $(12 / 12,100 \%)$ isolates carried $c p h A$, while none of 15 A. caviae isolates did. Four ( $80 \%)$ of five A. hydrophila isolates and three Aeromonas species had cphA.

With the exception of A. aquariorum A2-155, the DNA sequences of PCR products of $A$. aquariorum $\mathrm{MDC} 47^{\mathrm{T}}$ and A2-1000804, A. veronii bv. sobria ATCC $9071^{\mathrm{T}}, A$. hydrophila ATCC $7966^{\mathrm{T}}$ and 34 blood isolates, around $720 \mathrm{bp}$, corresponded to that of the A. hydrophila AE036 cphA gene (GenBank accession no. X57102) with an identity of $93-95 \%$. The PCR product of A2-155 had an extra $219 \mathrm{nt}$ inserted sequence compared to A. hydrophila AE036 cphA genes (Fig. S1). The GenBank accession numbers for the sequences of $c p h A$-related genes of $A$. aquariorum $\mathrm{MDC} 47^{\mathrm{T}}$ and 10 published A. aquariorum blood isolates (with the exception of A2-155) are JF972618-JF972628.

\section{cphA and imipenem susceptibility}

The results of three phenotypic methods for imipenem susceptibility testing of reference strains and 51 blood isolates are shown in Tables 1, 2 and 3, respectively. Using the three methods of disc diffusion, Etest and agar dilution to study imipenem susceptibility among 35 isolates carrying $c p h A$, imipenem non-susceptibility was detected in one $(3 \%)$ isolate with the standard inoculum, while it was detected among $12(34 \%), 17(49 \%)$ and 34 $(97 \%)$ isolates, respectively, with a large inoculum. Of note, $34 c p h A^{+}$isolates were resistant to imipenem (MICs $\geqslant 16 \mu \mathrm{g} \mathrm{ml}^{-1}$ ), as studied by the agar dilution test with a large inoculum, and were negative for $b l a_{\mathrm{IMP}}$ and $b l a_{\mathrm{VIM}}$ genes. By either the imipenem-EDTA combined disc test or MBL Etest, only $1(3 \%)$ of $34 c p h A^{+}$isolates exhibited an MBL-producing phenotype, while $10(29 \%)$ and 17 (49\%) isolates, respectively, did so with a large inoculum. None of $16 \mathrm{cph} \mathrm{A}^{-}$isolates exhibited imipenem nonsusceptibility or an MBL-producing phenotype with either a standard or large inoculum. Similarly, imipenem non-susceptibility in three $c p h A^{+}$reference strains was only demonstrated by the agar dilution test with a large inoculum.

Unlike most $c p h A^{+}$strains, two $c p h A^{+} A$. aquariorum isolates, A2-107 and A2-1000804, constantly exhibited imipenem non-susceptibility (MIC: 24 and $>32 \mu \mathrm{g} \mathrm{ml}{ }^{-1}$ by Etest with a standard inoculum, respectively) and expressed the MBL-producing phenotype by the imipenem-EDTA combined disc test and MBL Etest with either a standard or large inoculum. In contrast, one $c p h A^{+} A$. aquariorum isolate, A2-155, was always susceptible to imipenem by the three susceptibility tests with either inoculum. 


\section{MHT and imipenem susceptibility}

By the MHT, carbapenemase activity was detected among A. aquariorum $\mathrm{MDC}^{\mathrm{T}} \mathrm{T}^{\mathrm{T}}$ and $\mathrm{A} 2-1000804$, A. veronii bv. sobria ATCC $9071^{\mathrm{T}}$, A. hydrophila ATCC $7966^{\mathrm{T}}$ and 34 (97\%) of $35 c p h A^{+}$blood isolates, and was absent in $A$. caviae ATCC $13136^{\mathrm{T}}$ and $14(88 \%)$ of $16 \mathrm{cph} A^{-}$blood isolates (Fig. 1a-e). The MHT results for one $c p h A^{+}$ isolate (A. aquariorum A2-155) (Fig. 1f) and two $c p h A^{-} A$. caviae isolates were indeterminate because the indentation between the aeromonad streak and the margin of ertapenem inhibition zone was not obvious. The indeterminate MHT result for A2-155 was correlated with that of the agar dilution test with a large inoculum, in which imipenem non-susceptibility could not be demonstrated either. Overall, the MHT could detect carbapenemase activity in $34(100 \%)$ imipenem-nonsusceptible blood isolates.

\section{Clinical data}

Among 47 patients with Aeromonas bacteraemia from June 1999 to June 2001, only one patient had ever received carbapenem therapy. This patient with $c p h A^{+}$A. veronii and Enterobacter cloacae bacteraemia received an aminoglycoside active against both $E$. cloacae and $A$. veronii at bacteraemia onset and imipenem 1 week after bacteraemia onset and survived. The clinical condition of the patient with breakthrough bacteraemia due to A. aquariorum A21000804 during ertapenem therapy improved with ciprofloxacin therapy.

\section{DISCUSSION}

The conserved sequence of the $r p o B$ gene, encoding the $\beta$ subunit of the DNA-dependent RNA polymerase in the genus Aeromonas, has been used for delineating Aeromonas species (Küpfer et al., 2006; Lamy et al., 2010). Therefore, in the present study, the Aeromonas species were identified by, in addition to traditional biochemical reactions, sequence analyses of the partial $r p o B$ genes. We found that A. aquariorum was a predominant species causing bacteraemia. Such a fact highlights the potential clinical impact of $A$. aquariorium in, at least, southern Taiwan. A. aquariorum has been reported to be resistant to amoxicillin, cephalothin and cefoxitin (Figueras et al., 2009), but the corresponding types of $\beta$-lactamases have not been studied. Therefore, information on the $\beta$-lactamase profile of this clinically important species is valuable for rational recommendations for antimicrobial therapy and justifies the clinical relevance of the present study.

By PCR and the colony hybridization technique, the distribution of $c p h A$-related genes 93-95\% identical to $A$. hydrophila cphA genes (GenBank accession no. X57102) varied among Aeromonas species in the present study. $c p h A$-related genes were noted in A. veronii and A. hydrophila, but not in $A$. caviae. Such results were concordant 


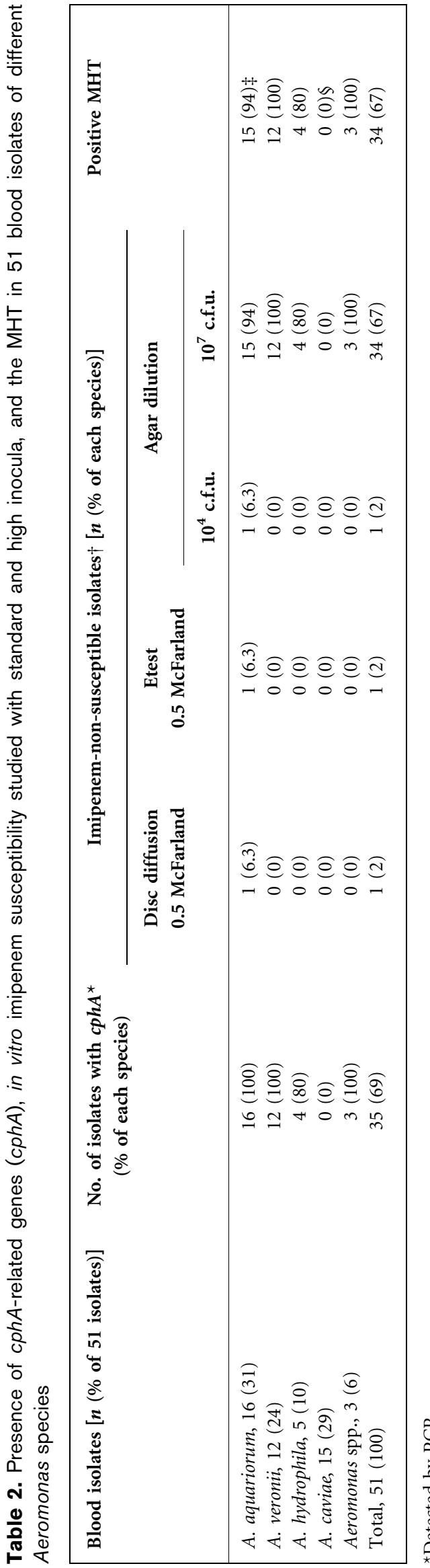

with those in previous studies (Balsalobre et al., 2009; Rossolini et al., 1995). However, it is a novel finding that the cphA-related genes were present in all A. aquariorum isolates.

Determination of in vitro susceptibility to carbapenems by either the disc diffusion or dilution techniques among carbapenemase-producing Aeromonas isolates has often failed to yield a carbapenem-resistant phenotype (Massidda et al., 1991; Rossolini et al., 1996). Likewise, our $c p h A^{+}$ aeromonads rarely exhibited the imipenem-non-susceptible phenotype by the disc diffusion, Etest or agar dilution techniques with the recommended inoculum. However, with a higher inoculum, imipenem non-susceptibility was increasingly demonstrated among $c p h A^{+}$isolates, and absent among $c p h A^{-}$isolates. Among the detection methods for carbapenem resistance, the agar dilution test with large inocula was the most sensitive $(97 \%)$. The only exception, A. aquariorum A2-155, was susceptible to imipenem even when tested with a large inoculum. Rossolini et al. (1995) found that $20 \%$ of cphA hybridized-positive $A$. hydrophila and $A$. veronii bv. sobria isolates were unable to express MBL activity, and they proposed that genetic modification had led to silencing of cphA in these species. Such a genetic modification was noted in A2-155, in which an extra inserted sequence in cphA might have altered the expression of CphA MBL. Owing to the failure of conventional in vitro susceptibility tests to detect carbapenemase production in aeromonads, we echo the advice of Rossolini et al. (1996) that it is prudent to consider Aeromonas species with carbapenemase-encoding genes as being resistant to carbapenems, unless they are verified to be unable to produce carbapenemase or to be carbapenem-susceptible by susceptibility testing using a large inoculum.

Similarly, the detection of cphA MBL activity, which could be inhibited by ion chelators, among aeromonads is inoculum-dependent. With the disc diffusion method and Etest, the zone diameters of inhibition increased and the MIC values decreased in the presence of EDTA among most $c p h A^{+}$isolates when tested with large inocula. Other phenotypic methods to detect MBL are the MHT (Lee et al., 2001) and synergy tests based on the ability of metal chelators (Arakawa et al., 2000). However, even with large inocula, EDTA synergy disc tests and Etest detected MBL among only one-third and one-half, respectively, of $c p h A^{+}$ aeromonads in our study. The MHT is recommended for the detection of carbapenemases in members of the Enterobacteriaceae by the CLSI (CLSI, 2009). However, the sensitivity and specificity of the test for detecting isolates with low-level carbapenemase production, such as aeromonads, are not known (CLSI, 2009). In our observation, the MHT performed well at detecting carbapenemase activity in our Aeromonas isolates.

The clinical relevance of CphA MBL in Aeromonas species remains obscure. In the literature, the clinical outcome in patients receiving carbapenems for infections caused by 
Table 3. Imipenem susceptibility, expression of MBL and the MHT in 51 Aeromonas blood isolates with or without $c p h A$-related genes

\begin{tabular}{|c|c|c|}
\hline \multirow[t]{2}{*}{ Method (inoculum) } & \multicolumn{2}{|c|}{ No. of isolates $(\%)$} \\
\hline & $\operatorname{cph} A^{+}(n=35)$ & $\operatorname{cph} A^{-}(n=16)$ \\
\hline \multicolumn{3}{|l|}{ Imipenem non-susceptibility ${ }^{\star}$} \\
\hline \multicolumn{3}{|l|}{ Standard inocula } \\
\hline Disc diffusion (0.5 McFarland) & $1(3)$ & $0(0)$ \\
\hline Etest (0.5 McFarland) & $1(3)$ & $0(0)$ \\
\hline Disc diffusion (1.5 McFarland) & $12(34)$ & $0(0)$ \\
\hline Etest (1.5 McFarland) & $17(49)$ & $0(0)$ \\
\hline Agar dilution ( $10^{7}$ c.f.u.) & $34(97)$ & $0(0)$ \\
\hline \multicolumn{3}{|l|}{ Phenotypic expression of MBL } \\
\hline Imipenem-EDTA combined disc & $10(29)$ & $0(0)$ \\
\hline Imipenem-EDTA MBL Etest & $17(49)$ & $0(0)$ \\
\hline Positive MHT with ertapenem disc & $34(97)$ & $0(0)$ \\
\hline
\end{tabular}

${ }^{\star}$ Imipenem non-susceptibility: an inhibition zone diameter $<16 \mathrm{~mm}$ by disc diffusion test or an MIC $>4 \mu \mathrm{g} \mathrm{ml}{ }^{-1}$ by Etest or agar dilution method.

(a)

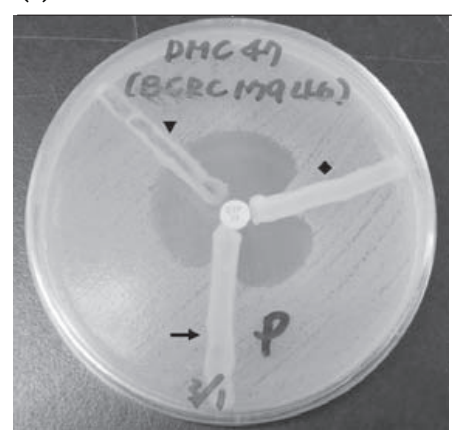

(d)

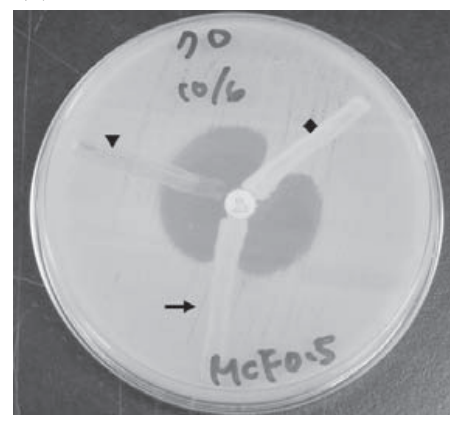

(b)

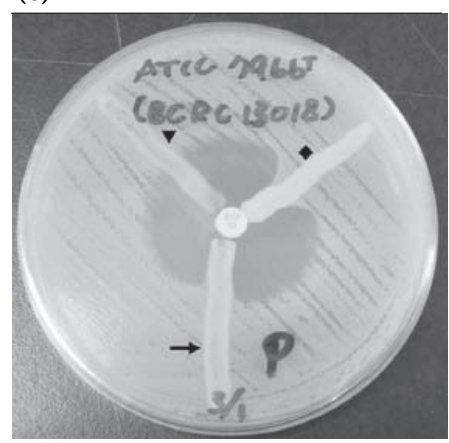

(e)

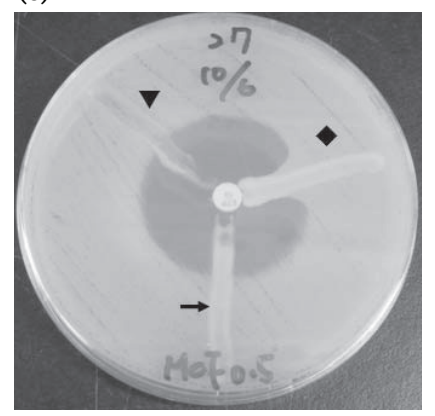

(c)

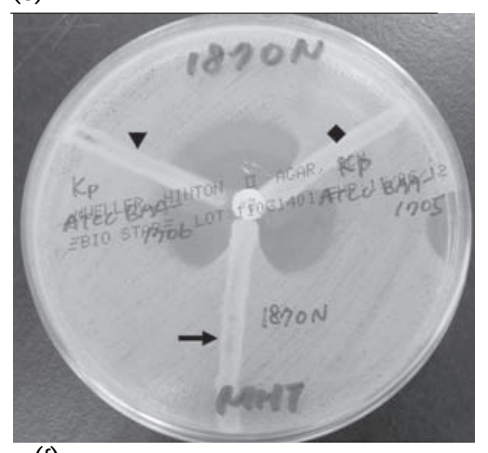

(f)

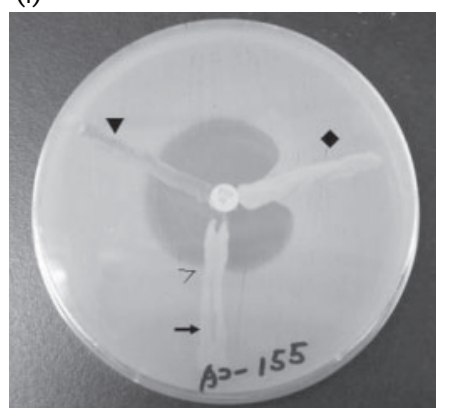

Fig. 1. Results of the MHT. Positive results for (a) A. aquariorum MDC47 ${ }^{\top}$, (b) A. hydrophila ATCC $7966^{\top}$, (c) $A$. aquariorum A2-1000804 and (d) A. aquariorum A2-70; (e) a negative result for $A$. caviae A2-27; (f) an indeterminate result for $A$. aquariorum A2-155, in which the indentation $(>)$ between the aeromonad streak and the margin of ertapenem inhibition zone was not obvious. $\rightarrow$, Tested Aeromonas isolate, $\bullet$ Klebsiella pneumoniae ATCC BAA-1705 as the positive control; $\mathbf{\nabla}, K$. pneumoniae ATCC BAA-1706 as the negative control; Escherichia coli ATCC 25922 was the indicator organism. 
Aeromonas species with cphA has been rarely described, and both successful (Mukhopadhyay et al., 2003) and fatal (Itoh et al., 1999) outcomes have been reported. Theoretically, carbapenem monotherapy would fail to inhibit the growth of MBL-producing aeromonads in infectious diseases with high tissue bacterial burdens, such as peritonitis/abdominal sepsis or soft tissue infections. The bacterial loads of these infected sites might reach more than $10^{7}$ c.f.u. $\mathrm{ml}^{-1}$ (Brian \& Julian, 2011; Mouës et al., 2004), much higher than the inoculum of $10^{4}$ c.f.u. $\mathrm{ml}^{-1}$ for the standard agar dilution test. Moreover, the production of CphA was significantly increased in the presence of a suitable $\beta$-lactamase inducer, such as benzylpenicillin or imipenem (Segatore et al., 1993). The emergence of an imipenem-resistant Aeromonas isolate during carbapenem treatment was noted in one of our patients and in a cirrhotic patient with A. hydrophila osteomyelitis (Lee et al., 2003). The development of imipenem resistance in an A. veronii isolate with ImiS ( $98 \%$ identical to CphA) recovered from a patient with antecedent amoxicillinclavulanate treatment has also been reported (SánchezCéspedes et al., 2009). These observations highlight the controversy of carbapenem therapy for infectious diseases caused by $c p h A$-carrying Aeromonas isolates. Therefore, we advise performing the susceptibility tests with a large inoculum or the MHT before considering a carbapenembased chemotherapy for infections due to Aeromonas species.

In conclusion, the distribution of $c p h A$-related genes among aeromonads is species-specific, found in A. aquariorum, A. veronii and A. hydrophila. However, $c p h A^{+}$Aeromonas species rarely exhibited the imipenem-non-susceptible phenotype using in vitro susceptibility tests unless they were tested with a large inoculum. The MHT could be a screening test for CphA production in aeromonads before a carbapenem-based chemotherapy.

\section{ACKNOWLEDGEMENTS}

This study was supported by the National Science Council, Taiwan (grants NSC 99-2628-B-006-014-MY3 and NSC 98-2320-B-006029); Department of Health, Executive Yuan (DOH100-TD-B-111002); and the National Health Research Institutes (id-100-pp-17). We thank Miss Hui-Ju Hung and Pei-Chen Wu for the laboratory work.

\section{REFERENCES}

Arakawa, Y., Shibata, N., Shibayama, K., Kurokawa, H., Yagi, T., Fujiwara, H. \& Goto, M. (2000). Convenient test for screening metallo$\beta$-lactamase-producing gram-negative bacteria by using thiol compounds. J Clin Microbiol 38, 40-43.

Aravena-Román, M., Harnett, G. B., Riley, T. V., Inglis, T. J. \& Chang, B. J. (2011). Aeromonas aquariorum is widely distributed in clinical and environmental specimens and can be misidentified as Aeromonas hydrophila. J Clin Microbiol 49, 3006-3008.

Balsalobre, L. C., Dropa, M., Lincopan, N., Mamizuka, E. M., Matté, G. R. \& Matté, M. H. (2009). Detection of metallo- $\beta$-lactamases-encoding genes in environmental isolates of Aeromonas hydrophila and Aeromonas jandaei. Lett Appl Microbiol 49, 142-145.

Brian, J. D. \& Julian, K. (2011). Medscape: Drug, Diseases \& Procedures. Peritonitis and Abdominal Sepsis. Available at http:// emedicine.medscape.com/article/180234-overview\#a0104. Updated on March 29, 2011.

CLSI (2009). Performance Standards for Antimicrobial Susceptibility Testing, 19th Informational Supplement. M100-S19. Wayne, PA: Clinical and Laboratory Standards Institute.

CLSI (2010). Methods for Antimicrobial Dilution and Disk Susceptibility Testing of Infrequently Isolated or Fastidious Bacteria; Approved Guideline M45-A2. Wayne, PA: Clinical and Laboratory Standards Institute.

Figueras, M. J., Alperi, A., Saavedra, M. J., Ko, W. C., Gonzalo, N., Navarro, M. \& Martínez-Murcia, A. J. (2009). Clinical relevance of the recently described species Aeromonas aquariorum. J Clin Microbiol 47, 3742-3746.

Fosse, T., Giraud-Morin, C. \& Madinier, I. (2003). [Phenotypes of beta-lactam resistance in the genus Aeromonas]. Pathol Biol (Paris) 51, 290-296 (in French).

Itoh, H., Kuwata, G., Tateyama, S., Yamashita, K., Inoue, T., Kataoka, H., Ido, A., Ogata, K., Takasaki, M. \& other authors (1999). Aeromonas sobria infection with severe soft tissue damage and segmental necrotizing gastroenteritis in a patient with alcoholic liver cirrhosis. Pathol Int 49, 541-546.

Janda, J. M. \& Abbott, S. L. (2010). The genus Aeromonas: taxonomy, pathogenicity, and infection. Clin Microbiol Rev 23, 35-73.

Küpfer, M., Kuhnert, P., Korczak, B. M., Peduzzi, R. \& Demarta, A. (2006). Genetic relationships of Aeromonas strains inferred from $16 \mathrm{~S}$ rRNA, gyrB and rpoB gene sequences. Int J Syst Evol Microbiol 56, 2743-2751.

Lamy, B., Laurent, F. \& Kodjo, A. (2010). Validation of a partial rpoB gene sequence as a tool for phylogenetic identification of aeromonads isolated from environmental sources. Can J Microbiol 56, 217-228.

Lee, K., Chong, Y., Shin, H. B., Kim, Y. A., Yong, D. \& Yum, J. H. (2001). Modified Hodge and EDTA-disk synergy tests to screen metallo- $\beta$-lactamase-producing strains of Pseudomonas and Acinetobacter species. Clin Microbiol Infect 7, 88-91.

Lee, C. H., Liu, M. S. \& Hsieh, S. H. (2003). Aeromonas hydrophila bacteremia presenting as non-traumatic acute osteomyelitis in a cirrhotic patient. Chang Gung Med J 26, 520-524.

Lee, C. C., Chi, C. H., Lee, N. Y., Lee, H. C., Chen, C. L., Chen, P. L., Chang, C. M., Wu, C. J., Ko, N. Y. \& other authors (2008). Necrotizing fasciitis in patients with liver cirrhosis: predominance of monomicrobial Gram-negative bacillary infections. Diagn Microbiol Infect Dis 62, 219-225.

Libisch, B., Giske, C. G., Kovács, B., Tóth, T. G. \& Füzi, M. (2008). Identification of the first VIM metallo- $\beta$-lactamase-producing multiresistant Aeromonas hydrophila strain. J Clin Microbiol 46, 1878-1880.

Martínez-Murcia, A. J., Saavedra, M. J., Mota, V. R., Maier, T., Stackebrandt, E. \& Cousin, S. (2008). Aeromonas aquariorum sp. nov., isolated from aquaria of ornamental fish. Int $J$ Syst Evol Microbiol 58, 1169-1175.

Massidda, O., Rossolini, G. M. \& Satta, G. (1991). The Aeromonas hydrophila cphA gene: molecular heterogeneity among class B metallo- $\beta$-lactamases. J Bacteriol 173, 4611-4617.

Mouës, C. M., Vos, M. C., van den Bemd, G. J., Stijnen, T. \& Hovius, S. E. (2004). Bacterial load in relation to vacuum-assisted closure wound therapy: a prospective randomized trial. Wound Repair Regen 12, 11-17. 
Mukhopadhyay, C., Bhargava, A. \& Ayyagari, A. (2003). Aeromonas hydrophila and aspiration pneumonia: a diverse presentation. Yonsei Med J 44, 1087-1090.

Neuwirth, C., Siebor, E., Robin, F. \& Bonnet, R. (2007). First occurrence of an IMP metallo- $\beta$-lactamase in Aeromonas caviae: IMP19 in an isolate from France. Antimicrob Agents Chemother 51, 44864488.

Rossolini, G. M., Zanchi, A., Chiesurin, A., Amicosante, G., Satta, G. \& Guglielmetti, P. (1995). Distribution of $c p h A$ or related carbapenemase-encoding genes and production of carbapenemase activity in members of the genus Aeromonas. Antimicrob Agents Chemother 39, 346-349.

Rossolini, G. M., Walsh, T. \& Amicosante, G. (1996). The Aeromonas metallo- $\beta$-lactamases: genetics, enzymology, and contribution to drug resistance. Microb Drug Resist 2, 245-252.

Sánchez-Céspedes, J., Figueras, M. J., Aspiroz, C., Aldea, M. J., Toledo, M., Alperi, A., Marco, F. \& Vila, J. (2009). Development of imipenem resistance in an Aeromonas veronii biovar sobria clinical isolate recovered from a patient with cholangitis. J Med Microbiol 58, 451-455.

Segatore, B., Massidda, O., Satta, G., Setacci, D. \& Amicosante, G. (1993). High specificity of $c p h A$-encoded metallo- $\beta$-lactamase from Aeromonas hydrophila AE036 for carbapenems and its contribution to $\beta$-lactam resistance. Antimicrob Agents Chemother 37, 1324-1328.
Talavera, B. M. M., Benassi, F. O., von Specht, M. H., Quiroga, M. I., Garcia, M. A., Pucciarelli, A. B., Zubreski, E., Laczeski, M. E. \& Gutkind, G. (2006). Susceptibilities to carbapenems and presence of cphA gene on food-borne Aeromonas. Braz Arch Biol Technol 49, 677682.

Walsh, T. R., Neville, W. A., Haran, M. H., Tolson, D., Payne, D. J., Bateson, J. H., MacGowan, A. P. \& Bennett, P. M. (1998). Nucleotide and amino acid sequences of the metallo- $\beta$-lactamase, ImiS, from Aeromonas veronii bv. sobria. Antimicrob Agents Chemother 42, 436439.

Wu, C. J., Wu, J. J., Yan, J. J., Lee, H. C., Lee, N. Y., Chang, C. M., Shih, H. I., Wu, H. M., Wang, L. R. \& Ko, W. C. (2007). Clinical significance and distribution of putative virulence markers of 116 consecutive clinical Aeromonas isolates in southern Taiwan. J Infect 54, 151-158.

Wu, C. J., Lee, H. C., Chang, T. T., Chen, C. Y., Lee, N. Y., Chang, C. M., Sheu, B. S., Cheng, P. N., Shih, H. I. \& Ko, W. C. (2009). Aeromonas spontaneous bacterial peritonitis: a highly fatal infectious disease in patients with advanced liver cirrhosis. J Formos Med Assoc 108, 293300.

Yong, D., Lee, K., Yum, J. H., Shin, H. B., Rossolini, G. M. \& Chong, Y. (2002). Imipenem-EDTA disk method for differentiation of metallo$\beta$-lactamase-producing clinical isolates of Pseudomonas spp. and Acinetobacter spp. J Clin Microbiol 40, 3798-3801. 